\title{
Trustworthy Privacy-Preserving Car-Generated Announcements in Vehicular Ad Hoc Networks
}

\author{
Vanesa Daza, Josep Domingo-Ferrer, Senior Member, IEEE, Francesc Sebé and Alexandre Viejo
}

\begin{abstract}
Vehicular ad hoc networks (VANETs) allow vehicleto-vehicle communication and, in particular, vehicle-generated announcements. Provided that the trustworthiness of such announcements can be guaranteed, they can greatly increase the safety of driving. A new system for vehicle-generated announcements is presented that is secure against external and internal attackers attempting to send fake messages. Internal attacks are thwarted by using an endorsement mechanism based on threshold signatures. Our system outperforms previous proposals in message length and computational cost. Three different privacypreserving variants of the system are also described to ensure that vehicles volunteering to generate and/or endorse trustworthy announcements do not have to sacrifice their privacy.
\end{abstract}

Index Terms-Vehicular communications, Security, Privacy, Secret sharing, Threshold signatures, Protocol design.

\section{INTRODUCTION}

A mobile ad hoc network (MANET) is formed by mobile nodes that are connected in a self-organized way without an underlying hierarchical infrastructure. In the special case where the mobile nodes are embedded in vehicles, the MANET is called vehicular ad hoc network (VANET).

VANETs permit a vehicle to automatically warn nearby vehicles about its movements (braking, lane change, etc.) to avert dangerous situations. These alert messages only require a limited dissemination (less than a hundred meters) but have very strong real-time requirements (they must be processed very quickly).

VANETs also allow a car to send announcement messages about road conditions (traffic jams, accidents) to other vehicles so that the latter can take advantage of that information to select routes avoiding troublesome points. Such announcement messages require a longer dissemination range. However, their requirement of real-time processing is much less strict than in the case of alerts, so that advanced cryptography can be used to make such messages secure and trustworthy. This paper focuses on announcements.

Privacy is a key aspect in vehicular ad hoc networks. The fact that a vehicle is equipped with communication capabilities should not render profiling its driver's habits (locations visited, driving pattern, etc.) any easier. Indeed, as noted in [1] a lot can be inferred on the driver's personality if the whereabouts and the driving pattern of a car can be tracked. There are two layers of privacy: anonymity and unlinkability. A system

Rovira i Virgili University, Department of Computer Engineering and Mathematics, UNESCO Chair in Data Privacy, Av. Països Catalans 26, E-43007 Tarragona, Catalonia, e-mail: \{vanesa.daza, josep.domingo, francesc.sebe, alexandre.viejo\}@urv.cat

Manuscript received XXX; revised XXX. preserves anonymity when it does not require the identity of its users to be disclosed. Unlinkability is stronger than anonymity and refers to the fact that different interactions of the same user with the system cannot be related. Unlinkability prevents user tracking and profiling.

Security in car-generated announcements sent over a VANET is fundamental. It is particularly important that the system does not permit an intruder (external attacker) or a dishonest driver (internal attacker) to attack integrity by either inserting fake announcements or modifying announcements sent by others. Tampered announcements could seriously disrupt traffic or cause dangerous situations for other vehicles.

Security against insertion of fake announcements by external attackers is easy to achieve using well-known cryptographic authentication techniques (digital signatures or message authentication codes). Such techniques require the sender of a message to access some secret key material only available to legitimate, registered users - and therefore unavailable to external attackers.

Dealing with internal attackers is a thornier issue. The reason is that legitimate system users, and thus internal attackers, have access to the secret key material required to send authenticated fake messages (for instance, to announce a false traffic jam with the aim of diverting traffic from a certain area where some kind of crime is being committed). Countermeasures against fake messages from internal attackers fall into two classes: a posteriori and a priori.

\section{A. A posteriori countermeasures}

A posteriori countermeasures consist of taking punitive actions against users who have been proven to have originated fake messages (e.g. the offenders can be banished from the network). These countermeasures in anonymous systems require the presence of a trusted third party able to revoke the key material of such dishonest users. In this way, they will be excluded from the system.

Digital signatures have been extensively used in most of the protocols that offer a posteriori countermeasures: from plain digital signatures [2], [3], [4], [5] until more sophisticated distributed signatures, such as group signatures in [6] or ring signatures in [7]. The latter paper and [8] also consider IDbased ring signatures.

\section{B. A priori countermeasures}

A priori countermeasures attempt to prevent the generation of fake messages. In this approach, a message is not considered valid unless it has been endorsed by a number of vehicles 
above a certain threshold. Those vehicles must be in a position to confirm what is reported in the message: for a traffic jam announcement, other jammed vehicles are potential endorsers (automatically or after intervention of their drivers); for an "icy road" message, nearby vehicles whose traction system has detected slippery ground can be automatic endorsers. This approach is based on the assumption that most users are honest so that they will not endorse any message containing false data.

Under this approach, the risk that a collusion of dishonest vehicles reaches the size necessary to generate fake messages always exists. The natural strategy against collusions is to choose a threshold sufficiently high so as to render successful collusions unlikely. However, this threshold should not be so high that it prevents honest vehicles from sending true announcements in situations with a low density of vehicles.

The a priori approach is compatible with driver privacy: since false announcements are thwarted without resorting to punitive actions, unconditional vehicle anonymity is allowable (in contrast, a posteriori countermeasures assume that offenders are identified and punished).

The use of a honest majority to prevent generation of fake messages has previously been proposed in [9], [10], [11], [12]. A brief discussion of those papers is next given.

In [9] a framework is presented to validate received data in VANETs. In this approach, a vehicle receives alerts from different neighbors and compares them in order to infer the correctness of a certain event. This scheme suffers from high communication overhead due to the lack of aggregation techniques. Besides, the proposed framework has not been empirically tested. The paper [10] presents a contribution that remains quite vague: VANET security issues are identified, some security primitives are enumerated, but no complete protocol is actually described. In [11], a system that evaluates the plausibility of received danger warnings is proposed. This system estimates the trustworthiness of a reported hazard by taking a vote on the received danger messages. The paper provides a simulative analysis of different voting schemes, but privacy remains unaddressed and security is not completely covered. Finally, [12] describes a detailed protocol deployable in real VANET environments (the authors show this via simulation) which systematically deals with security threats and reduces communication overhead by aggregating messages.

According to the above discussion, [12] seems the most competitive scheme in the literature on the a priori approach, so we concentrate on it in what follows. That paper presents three variants offering a priori countermeasures against fake messages: concatenated signatures, onion signatures and hybrid signatures.

In the variant based on concatenated signatures, a vehicle generates an announcement and sends it, its signature and its public-key certificate to a nearby car which will endorse it by computing its own signature on it. This new signature and the corresponding public-key certificate will be appended to the frame that will be retransmitted to the next vehicle. An announcement is considered valid after it has been endorsed by at least the number of vehicles determined by the threshold. This approach has several drawbacks:

- It does not offer unlinkability since different signatures made by the same user can be linked through the public key that verifies them. Anonymity is however feasible by using pseudonyms.

- Announcement generation is delayed due to the sequential communication pattern (the delay is proportional to the number of endorsing vehicles).

- It requires the verifier to check several signatures upon receiving an announcement (as many verifications as vehicles have endorsed the message). These verifications involve checking the validity of public-key certificates and probably revocation lists as well.

Therefore, there is room for improvement both in terms of privacy and efficiency (communication and computation costs).

The variants based on onion signatures and hybrid signatures are similar and designed to reduce the overall message length. Both variants use the so-called oversignatures: instead of appending its signature, each new endorsing car signs the signature by the previous endorsing car (this is called oversigning). In an oversignature, a verifier can check the last endorser's signature, but not the signatures by the previous endorsers. Since this is a serious design flaw, we will only consider the concatenated signatures variant for comparison in the rest of this paper.

\section{Contribution and plan of this paper}

In this work, a proposal is presented following the $a$ priori protection paradigm that reduces the verification cost of endorsed messages to one signature verification. Three different privacy-preserving variants of the system are also described to ensure that vehicles volunteering to generate and/or endorse trustworthy announcements do not have to sacrifice their privacy (anonymity and unlinkability). Section II gives some brief background on secret sharing and threshold signatures. Section III presents the new protocol and three privacy-preserving variants of it, which are eventually combined in a compound protocol. Section IV studies the proposed protocol using simulations. Finally, Section V contains some concluding remarks.

\section{BACKGROUND}

This section gives some minimalistic cryptographic background needed to understand the rest of the paper.

\section{A. Secret sharing}

A secret sharing scheme is a method by means of which a special figure, called dealer, distributes a secret $s$ among a set $\mathcal{P}=\left\{P_{1}, \ldots, P_{n}\right\}$ of $n$ players. The dealer secretly sends to each player $P_{i}$ his share $s_{i}$ of the secret $s$ in such a way that only authorized subsets can recover the secret.

A $(t, n)$-threshold secret sharing scheme is a particular case in which authorized subsets are those composed of at least $t$ players. Shamir's threshold secret sharing scheme [13] gives a solution to this problem. Indeed, let $\mathbb{Z}_{q}$ be a finite field with $q>n$ and $s \in \mathbb{Z}_{q}$ be the secret to be shared. The dealer picks a polynomial $p(x)$ of degree at most $t-1$ at random, whose 
free term is the secret $s$, that is, $p(0)=s$. The polynomial $p(x)$ can be written as $p(x)=s+\sum_{j=1}^{t-1} a_{j} x^{j}$, where $a_{j} \in \mathbb{Z}_{q}$ has been randomly chosen.

Each player $P_{i}$ is assigned a known value $\alpha_{i} \in \mathbb{Z}_{q}$. Then, the dealer privately sends to player $P_{i}$ his share $s_{i}=p\left(\alpha_{i}\right)$, for $i=1, \ldots, n$.

Therefore, a set $A \subset \mathcal{P}$ of at least $t$ players can recover the secret $s=p(0)$ by interpolating the set of shares they hold:

$$
s=p(0)=\sum_{P_{i} \in A} s_{i} \lambda_{i}^{A}=\sum_{P_{i} \in A} s_{i}\left(\prod_{P_{j} \in\left(A \backslash P_{i}\right)} \frac{-\alpha_{j}}{\alpha_{i}-\alpha_{j}}\right)
$$

Values $\lambda_{i}^{A}$ are called the Lagrange coefficients. It can be proven that less than $t$ players cannot obtain any information about the secret $s$.

\section{B. Threshold signatures}

Digital signatures allow to send authenticated and nonrepudiable messages. The message sender is required to have a public/private key pair. Signature generation is an algorithm that takes as input the message, $m$, and the sender's private key, $S K$. Its output is the signature $\sigma(m)$ on $m$. Signature verification is performed by the receiver. Its algorithm takes as input the message $m$, its signature $\sigma(m)$ and the sender's public key $P K$. It outputs "yes" or "no" to reflect the validity of $\sigma(m)$. A valid signature convinces the receiver about the integrity of $m$ and is taken as a proof that the message was generated by the authentic sender (the only party knowing $S K)$.

A $(t, n)$-threshold signature distributes the signing operation among a group of $n$ participants. Each participant in a distributed signature scheme is given a share, $S K_{i}$, of the secret key, $S K$, in such a way that to sign a message every participant computes a partial signature, $\sigma_{i}(m)$, using his share of the secret key. Then, any set of at least $t$ participants can compute a valid signature $\sigma(m)$ on the message by combining their partial signatures. The resulting signature is equivalent to the one that results in the non-distributed case (it is also verifiable using $P K$ ). A distributed signature scheme is said to be non-interactive if every participant can compute his partial signature on a message $m$ without interacting with the rest of participants. Signatures in [14], [15], [16], [17] are examples of non-interactive threshold signature schemes.

For the sake of concreteness, we next recall an efficient threshold signature scheme, namely the one in [15], a distributed version of the signature scheme by Boneh, Lynn and Shacham (BLS, [18]). Both schemes work over Gap DiffieHellman (GDH) groups - see original papers for more details. In a nutshell, these signature protocols based on pairings are quite efficient as the signing process only requires hash operations and modular exponentiations and the verification process two pairing computations. In [19] a fast implementation of the Tate pairing computation was given and the BLS signature scheme was compared with an RSA signature on a Pentium PIII processor at $1 \mathrm{GHz}$. Using RSA with a modulus length $|n|=1024$ bits and a private exponent length $|d|=1007$ bits, signing took $7.90 \mathrm{~ms}$ and verifying took $0.4 \mathrm{~ms}$. Using the
BLS signature with elliptic curves over $\mathbb{F}_{3^{97}}$, signatures were 160 bits long (which yields a similar security as the abovementioned 1024-bit RSA signature), and signing and verifying took $3.57 \mathrm{~ms}$ and $53 \mathrm{~ms}$, respectively. So there exist threshold signatures with reasonable computational cost.

Let $\mathbb{G}$ be a GDH group, $g=<\mathbb{G}>$ be a generator of the group and $p$ be the order of the group. Using methods described in [20], every participant $P_{i}$ obtains a share $S K_{i}$. The set of shares realizes a $(t, n)$-threshold access structure, that is, $t$ parties can retrieve the secret key $S K$ whereas less than $t$ cannot obtain any information on the secret key. The retrieval process can be performed by means of Lagrange interpolation and also yields the matching public key $P K=g^{S K}$. To sign a message $m$, a participant $P_{i}$ computes his partial signature as $\sigma_{i}(m)=\mathcal{H}(m)^{S K_{i}}(\mathcal{H}$ is a public one-way and collision-free hash function whose input is a string of arbitrary length and whose output is an element in $\mathbb{G} \backslash\{1\}$ ) and broadcasts $\sigma_{i}(m)$. After a set $A$ of at least $t$ participants have broadcast their partial signatures $\sigma_{i}(m)$ for message $m$, a standard signature $\sigma$ for the message can be computed as

$$
\sigma(m)=\prod_{i \in A} \sigma_{i}(m)^{\lambda_{i}^{A}}=\mathcal{H}(m)^{\sum_{i \in A} \lambda_{i}^{A} S K_{i}}=\mathcal{H}(m)^{S K}
$$

where $\lambda_{i}^{A}$ are the Lagrange coefficients.

\section{Privacy in secret sharing}

In short, an anonymous secret sharing scheme is one where participants can co-operate in the retrieval of the secret while keeping their identity undisclosed (anonymity) and without successive co-operations by the same participant being linkable (unlinkability). Shamir's $(t, n)$-threshold secret sharing scheme described in Section II-A does not offer unlinkability: each Lagrange coefficient corresponds to a certain participant $P_{i}$ and, even if that correspondence is kept secret for anonymity (i.e. by using the underlying $\alpha_{i}$ as pseudonyms), successive co-operations by the same participant can be linked because the Lagrange coefficient of the participant appears every time. Anonymous secret sharing schemes in the literature present a very high cost that limits their practical applicability [21].

Note that, if the secret sharing scheme underpinning a threshold signature protocol is not anonymous, the resulting threshold signature is either linkable (successive partial signatures by a participant can be linked) or requires a trusted third-party and is thus unsuitable for a VANET.

\section{OUR PROPOSAL}

In this section a new system for secure announcements in VANETs is presented. It uses digital signatures to prevent external attackers from being able to inject false messages and follows the a priori approach to thwart fake announcements sent by internal attackers. An announcement will only be considered valid if it has been endorsed by at least $t$ different vehicles. 


\section{A. Non-private protocol}

For clarity, let us begin with a protocol which can offer anonymity but not unlinkability.

- Set-up: During this stage, the carmakers set up a $(t, n)$ threshold signature scheme, where $n$ is the maximum number of vehicles allowable in the VANET. To do this, the carmakers must agree on a polynomial of degree $(t-1)$ that will be evaluated at points $\alpha_{i}$, for $i=1$ to $n$. The range of $n$ points is partitioned into several subranges, each of which is assigned to a carmaker. The number $n$ can be very large without scalability problems. Next, a public key $P K$ and $n$ shares $S K_{i}, i=1, \ldots, n$ of the secret key $S K$ are generated. Each vehicle $P_{i}$ is equipped with the public key $P K$ and its secret key share $S K_{i}$; the share $S K_{i}$ is held in a smart card plugged into the vehicle (tamper-resistance is assumed for the card in what follows). When input the hash value $\mathcal{H}(m)$ of a message, the smart card returns a partial signature on that hash value, that is, $\sigma_{i}(m)=\mathcal{H}(m)^{S K_{i}}$. Anonymity is obtained by not linking $S K_{i}$ with the identity of the vehicle; this makes sense for other reasons too because, smart cards being removable, several smart cards each holding a different secret key share could alternatively be used with the same vehicle (like several cards can be used with a cellphone).

- Announcement generation: When a vehicle $P_{i}$ wishes to send an announcement $m, P_{i}$ computes the partial signature $\sigma_{i}(m)$ and broadcasts $m$ and $\sigma_{i}(m)$. An announcement should only reach vehicles that are close enough to the originating vehicle so as to be able to check the validity of the announced condition. Since they do not need to reach distant points, announcement messages are not relayed by VANET nodes and they travel only up to the range of the broadcast technology used (even if a maximum range of 1000 meters for car-to-car communication with the Dedicated Short Range Communication protocol is reported in [22], typical ranges from 300 to 500 meters on highways and about 100 meters in cities are mentioned in [23]).

- Announcement endorsement: If vehicle $P_{j}$ receives an announcement $m$ (together with the partial signature on it by the announcement originator $P_{i}$ ) and wishes to endorse $m$, then $P_{j}$ computes its own partial signature $\sigma_{j}(m)$ on $m$ and broadcasts $\mathcal{H}(m)$ and $\sigma_{j}(m)$ to return them to $P_{i}$, where $\mathcal{H}()$ is the same hash function used in the signature computation. As in announcement generation, messages with partial signatures are not relayed.

- Signature composition: The vehicle $P_{i}$ which generated an announcement stores $m$ and the partial signatures on $m$ it receives (partial signatures on $m$ are identifiable by the hash $\mathcal{H}(m)$ they carry). Once $P_{i}$ has collected $t$ different partial signatures on $m$, it can compute a standard signature $\sigma(m)$ and broadcast it along with $m$.

- Announcement reception and verification: Vehicles in the VANET will only consider as trustworthy those announcements carrying a standard signature that can be verified using the public key $P K$. The use of the threshold signature scheme provides vehicles with the assurance that such a standard signature can only have been computed if at least $t$ vehicles have endorsed $m$ by computing their partial signature on it. These messages, containing a standard signature, will be relayed by VANET nodes. In this way, they will reach distant vehicles which will benefit from the information in the messages.

The reason for keeping $S K_{i}$ in a smart card is to prevent the vehicle driver from learning $S K_{i}$; otherwise, $t$ colluding drivers could recover the secret key $S K$, which would allow any single one of them to sign messages that would be accepted as trustworthy without any endorsement.

In any case, the choice of $t$ is a trade-off between security and availability. On one hand, $t$ should be high enough so that the probability of there being $t$ or more within-range colluding vehicles who could validly endorse fake messages is reasonably low (security). On the other hand, $t$ should not be so high that finding $t-1$ additional within-range endorsers is too difficult for an honest announcement generator (availability).

The problem with the above protocol is that it lacks privacy and, more precisely, unlinkability. This is due to the fact that signature composition requires computing Lagrange coefficients (see Section II-A). Computation of such coefficients requires in turn knowledge of the value $\alpha_{i}$ assigned to each vehicle $P_{i}$ having contributed a partial signature. Certainly, it can be assumed and it is assumed that the correspondence between $P_{i}$ and $\alpha_{i}$ is withheld ( $\alpha_{i}$ is used a pseudonym for vehicle $P_{i}$ ), which provides anonymity. However, different partial signatures generated by the same vehicle $P_{i}$ all use $\alpha_{i}$, so they are linkable. Therefore, unlinkability is not achieved.

\section{B. Cost analysis of the non-private protocol}

In this section we compare the cost of our non-private protocol above with the cost of the concatenated signatures protocol in [12]. Both protocols are non-private, so the comparison is fair. In the next subsections, the cost is analyzed in terms of announcement length, announcement generation time and announcement verification time.

1) Announcement length: In the concatenated signatures protocol in [12], authenticated announcements contain as many signatures and public key certificates as endorsing vehicles, so their length is $O(t)$. In our proposal, both the partially signed announcements and the completely signed announcements contain a single signature, so the length of announcements is $O(1)$.

Since the above comparison in $O$-notation may be misleading for small values of $t$, we next compare both proposals by taking the constant terms into account. We assume that [12] uses the concatenated signatures protocol with the RSA public key cryptosystem with 1024 bit moduli (so, digital signatures will be 1024 bits long). Let us consider that the information which is announced is $a$ bits long. The concatenated signatures protocol in [12] requires one signature and one public key certificate from $t$ different signers. We will consider that a digital certificate contains an RSA public key (barely longer 
than the 1024-bit modulus if a short public exponent is used), the owner's pseudonym (which could be a 64-bit serial number) and a signature by the Certification Authority (1024 bits). According to that, the total length of an announcement in [12] is $a+t \cdot(3 \cdot 1024+64)$ bits. For example, if four endorsing vehicles are required $(t=4)$, this scheme yields an announcement length of $a+12544$ bits. With the same assumptions, our proposal has a constant announcement length of $a+160$ bits (we are using the BLS signature scheme). As $t$ grows, the advantage of using our system increases.

2) Announcement generation time: In [12] vehicles sequentially contribute with their signature to endorse an announcement. This means that a valid message generation takes at least the time necessary for a message to perform $t-1$ hops plus the time required to compute $t$ digital signatures. This is an $O(t)$ cost. Let $j$ be the time (in milliseconds) necessary for a message to perform one hop. According to the signature generation time reported in Section II-B, a valid message generation in [12] using the RSA cryptosystem with 1024 bit public keys takes $7.90 \cdot t+(t-1) \cdot j$ ms.

In our protocol, vehicles can endorse a message in parallel. So, the delay due to data transmission required to generate a valid message is fixed to the time to perform 2 hops (one from the generator to within-range endorsers and another from endorsers to the generator) plus the time to compute 2 BLS signatures. This time is $2 \cdot(j+3.57) \mathrm{ms}$.

After $t$ endorsement messages have been collected in our protocol, a standard signature is composed by the vehicle originating a message at an $O(t)$ cost (the cost of computing a standard signature from $t$ partial signatures). As can be seen in Section II-B, the cost of this operation is dominated by the exponentiation of each partial signature to its corresponding Lagrange coefficient. The cost of each exponentiation is similar to the cost of computing one digital signature (also consisting of one exponentiation). Thus, the composition time is approximately $t \cdot 3.57 \mathrm{~ms}$.

The overall generation time with our protocol is $2 \cdot(j+$ $3.57)+t \cdot 3.57 \mathrm{~ms}$. This expression can be rewritten as $2 \cdot j+(t+2) \cdot 3.57 \mathrm{~ms}$. This is a shorter time than the one required by [12]. As $t$ grows, the advantage of using our system increases.

3) Announcement verification time: In [12] announcement verification requires checking $t$ signatures and $t$ public key certificates. If certificates are subject to revocation, there is an additional cost related to checking certificate revocation lists (even this cost is not explicitly mentioned in [12]). In any case, the verification cost is $O(t)$.

In our protocol, an announcement is verified by checking one signature. Since the public key $P K$ used for verification is always the same and is stored in the smart card by the carmaker, its validity does not need to be checked. This is an $O(1)$ cost.

Let us now consider the constant terms for greater accuracy. Assume the RSA and the BLS signature schemes are used by [12] and our proposal, respectively. Section II-B details the signature verification time for each signature scheme. In this way, an announcement verification in [12] takes $2 \cdot 0.4 \cdot t$ $\mathrm{ms}$ (the verifier checks the certificate and message signatures
TABLE I

COST BREAKDOWN AS A FUNCTION OF THE THRESHOLD $t$ OF THE NON-PRIVATE PROTOCOL IN [12] AND THE NON-PRIVATE PROTOCOL IN THIS PAPER

\begin{tabular}{|l|c|c|}
\hline & Protocol [12] & Our protocol \\
\hline \hline Announcement length & $O(t)$ & $O(1)$ \\
Announcement generation time & $O(t)$ & $O(t)$ \\
Announcement verification time & $O(t)$ & $O(1)$ \\
\hline
\end{tabular}

sent by each endorsing vehicle). The same operation using our protocol takes $53 \mathrm{~ms}$. Therefore, with those assumptions, our proposal outperforms [12] only when $t \geq 67$. In practice, $t$ will be usually less than 67 , so that [12] will normally be faster than our protocol as far as the computation involved in signature verification goes.

Nonetheless, if the time and communication needed to check certificate revocation lists was taken into account, our proposal would be more efficient, because in [12] a certificate revocation list may need to be checked for each certificate to be verified.

4) Summary of cost analysis: Table I summarizes the cost of both protocols as a function of the threshold $t$. The strong points of our proposal are that the following is constant: announcement length and announcement verification time.

If a more accurate analysis of the constant terms is performed (which is necessary when $t$ is small), it turns out that our system still yields shorter announcements and faster announcement generation than [12]. Announcement verification, on the contrary, is faster with [12] at least for the usual (small) values of $t$.

However, if the cost of checking certificate revocation lists is considered in announcement verification, the picture changes dramatically. Indeed, [12] requires verifying $t$ certificates, which may require checking certificate revocation lists $t$ times. This may be very long, as it involves communication, not just computation. In our proposal, the validity of $P K$ does not need checking, as explained above. So, when the cost of checking certificate revocation is included, our proposal is more efficient also for announcement verification.

\section{Group-based private protocol}

In this section, a modification of the previous protocol is described in order to provide unlinkability. The modification mainly affects the set-up phase.

- Set-up: The $n$ vehicles that form the VANET are divided into $r$ groups, with each group consisting of $n / r$ vehicles (for simplicity, it is assumed that parameters $n$ and $r$ are chosen so that $r$ divides $n$, but suitable rounding can be used in the general case). The carmakers set up a $(t, r)$-threshold signature scheme. During this generation, a public key, $P K$, and $r$ shares, $S K_{j}, j=1, \ldots, r$, of the secret key $S K$ are generated (one share for each group). Each carmaker keeps a copy of each of the $r$ shares. Each manufactured vehicle $P_{i}$ is randomly assigned by the carmaker to a group $j$; then it is equipped with the public key $P K$ and the secret key share $S K_{j}$ assigned to its group (as above, $S K_{j}$ is held in a smart card plugged to the vehicle). 
This modification causes vehicles belonging to the same group to be assigned the same secret key share. In this way, partial signatures cannot be related to a single vehicle but to any member of its group. If groups are large enough, this protocol provides unlinkability. On the other side, a valid signature $\sigma(m)$ must now be generated not just by any $t$ vehicles, but by vehicles belonging to at least $t$ different groups.

1) Security, privacy and availability: Parameters $t$ and $r$ of the group-based protocol have an impact on security against fake messages, on privacy and on availability.

The threshold $t$ should be set high enough so that the probability of there being $t$ or more colluding vehicles who could validly endorse false announcements is reasonably low.

For a choice of $t$, parameter $r$ must be chosen considering the trade-off between unlinkability and availability:

- Unlinkability. The group size $g:=n / r$ must be large enough so that linkability at the group level (which cannot be avoided) does not imply linkability at the vehicle level.

- Availability. The number of groups $r$ must be large enough so that, given an announcement, finding $t$ endorsing vehicles from different groups is easy. Thus, $r \gg t$.

By construction, this proposal has the same cost as the nonprivate protocol (see Section III-A).

\section{Extended group-based private protocol}

In the previous group-based protocol, it may be difficult in some cases to find a value for $r$ striking a balance between unlinkability and availability. This is the case when the VANET is sparse or consists of an actual number $n^{\prime}$ of vehicles much less than the maximum allowable number $n$. Since the group size cannot be too small if unlinkability is to be preserved, the number $r$ of groups has to be small. In those conditions finding $t$ within-range endorsing vehicles from different groups may be quite challenging.

A solution to mitigate the problem caused by a small $r$ is to use $d$ different threshold signature schemes so that, if $t$ within-range endorsing vehicles from different groups cannot be found for the first scheme, they are sought for the second scheme, and so on. The modified set-up, announcement generation, endorsement and signature composition phases are:

- Set-up: The $n$ vehicles that form the VANET are divided into $r$ groups, as in Section III-C. The carmakers set up $d$ different $(t, r)$-threshold signature schemes. For $k=1$ to $d$, the $k$-th scheme consists of a public key $P K^{k}$ and $r$ shares, $S K_{j}^{k}, j=1, \ldots, r$ (one share per group). Each carmaker keeps a copy of all $r$ shares for all $d$ signature schemes. For $i=1, \ldots, n$, each manufactured vehicle $P_{i}$ is equipped with the public keys $\left(P K^{1}, \ldots, P K^{d}\right)$ and the secret key shares $\left(S K_{i_{1}}^{1}, \ldots, S K_{i_{d}}^{d}\right)$, where $i_{k} \in_{R}\{1, \ldots, r\}$ is the group randomly assigned by the carmaker to $P_{i}$ for the $k$-th threshold signature scheme. As above, all secret key shares are held in a smart card.

The only variation in the extended group-based protocol with respect to the previous protocols (non-private, groupbased) in what respects the announcement generation, endorsement and signature composition steps is that now messages in those steps must include a field specifying which threshold signature scheme among the $d$ possible ones is being used in a particular execution.

Announcement generation, endorsement and signature composition are attempted for the first threshold signature scheme as in Section III-C. If, after a predefined timeout, partial signatures from $t$ different groups have not been collected, announcement generation and endorsement are re-started for the second threshold signature scheme. The process stops when a threshold signature scheme is found for which endorsements from $t$ different groups can be collected. In the worst case, all $d$ threshold signatures schemes can fail.

Storage requirements at the vehicles are increased. In this case, each vehicle stores $d$ key shares and $d$ public keys (compared to one share and one public key in the previous proposal).

\section{E. Semi-private protocol for sparse VANETs}

The protocol in Section III-D is not without drawbacks. Even with $d$ different threshold signature schemes, collecting endorsement from $t$ different groups may fail in very sparse VANETs. A way to circumvent the above problem is to drop groups but to keep several threshold signature schemes for privacy. The modified protocol looks as follows:

- Set-up: The carmakers set up $d^{\prime}$ different $(t, n)$-threshold signature schemes. Like in the non-private protocol of Section III-A but for each signature scheme in this protocol, the range of $n$ points corresponding to possible vehicles is partitioned into several subranges, each of which is assigned to a carmaker. For $k=1$ to $d^{\prime}$, the $k$-th scheme consists of a public key $P K^{k}$ and $n$ shares, $S K_{i}^{k}, i=1, \ldots, n$ (one share per vehicle). For $i=1, \ldots, n$, each vehicle $P_{i}$ is equipped with the public keys $\left(P K^{1}, \ldots, P K^{d^{\prime}}\right)$ and the secret key shares $\left(S K_{i}^{1}, \ldots, S K_{i}^{d^{\prime}}\right)$, with share $S K_{i}^{k}$ being obtained by evaluating the polynomial of the $k$-th scheme at point $\alpha_{i}^{k}$, where $\alpha_{i}^{k}$ is assumed to belong to the subrange of the carmaker of $P_{i}$ for the $k$-th scheme.

- Announcement generation: When a vehicle $P_{i}$ wishes to send an announcement $m, P_{i}$ randomly selects one of the $d^{\prime}$ threshold signature schemes, say scheme $k$. One can assume that the selection is performed by the smart card in the vehicle so that the selected $k$ is beyond the user's control. Then $P_{i}$ computes its partial signature $\sigma_{i}^{k}(m)$ on $m$ and broadcasts the announcement and its partial signature. This solution also requires messages to include a field indicating which signature scheme $k$ is being used.

- Announcement endorsement: If vehicle $P_{j}$ receives the announcement $m$ (together with the partial signature on it by the announcement originator $P_{i}$ ) and wishes to endorse $m, P_{j}$ uses the $k$-th threshold scheme to compute its own partial signature $\sigma_{j}^{k}(m)$ on $m$ and broadcasts $\mathcal{H}(m)$ and $\sigma_{j}^{k}(m)$, where $\mathcal{H}()$ is the same hash function used in the signature computation.

- Signature composition: The vehicle $P_{i}$ which generated an announcement stores $m$ and the partial signatures on $m$ it receives (partial signatures on $m$ are identifiable 
by the hash $\mathcal{H}(m)$ they carry). Once $P_{i}$ has collected $t$ different partial signatures on $m$, it can compute a standard signature $\sigma^{k}(m)$ and broadcast it along with $m$.

- Announcement reception: Vehicles in the VANET will only consider as trustworthy those announcements carrying a standard signature that can be verified using a public key $P K^{k}$ in the set $\left(P K^{1}, \ldots, P K^{d^{\prime}}\right)$.

The above semi-private protocol requires vehicles to store $d^{\prime}$ shares and $d^{\prime}$ public keys.

1) Security, privacy and availability: As in the previous protocols, the threshold $t$ is the parameter controlling security against insertion of fake announcements.

Unlinkability is related to parameter $d^{\prime}$, the number of threshold signature schemes set up by the carmaker for this protocol. Provided that the threshold signature scheme is randomly selected, the probability that two successive participations by $P_{i}$ can be linked is $1 / d^{\prime}$ (this happens if the same threshold signature scheme is selected in both cases). Thus, unlinkability improves with respect to the non-private protocol (Section III-A) but it is worse than in the group-based or extended group-based protocols (Section III-C and III-D, respectively). However, the advantage is increased availability in that there are no constraints on the $t$ vehicles that must endorse an announcement (any $t$ vehicles will do), so that the endorsement process is easier in very sparse VANETs with really few vehicles per area unit.

A way to improve unlinkability is by taking a large $d^{\prime}$, which does not affect the announcement verification time. This is different from what happens in the extended groupbased protocol if parameter $d$ is increased: there, the signature schemes are tried one after the other until a valid signature is obtained or the $d$ schemes have been tried, so a large $d$ may result in longer verification times.

\section{F. Compound protocol}

The protocol in Section III-E can be used as a fallback for the protocol in Section III-D, which in turn is a fallback for the protocol in Section III-C. The idea is that vehicles can be set up for all three protocols by the carmaker. The first option to be tried is the group-based protocol. If traffic sparseness is such that partial signatures from $t$ different groups cannot be collected for a certain announcement before a fixed timeout, then the protocol in Section III-D is used. If this does not work either, the protocol Section III-E can be used to get limited unlinkability without increasing the difficulty of collecting endorsements with respect to the non-private protocol. According to that, a compound protocol combining the protocols in Sections III-C, III-D and III-E can be specified as follows:

1) Initially, the group-based protocol of Section III-C is used. Note that this protocol is a particular case of the extended group-based protocol where there is only one $(t, n)$-threshold signature scheme in use. Thus, hereafter we will consider this step as a part of the next step, where the extended group-based protocol is used. (In what follows we will only refer to the extended groupbased protocol and the semi-private protocol. This also applies to the simulation results which will be presented in Section IV.)

2) If a complete signature cannot be constructed before a certain timeout, the extended group-based system of Section III-D is launched. Construction of a complete signature by means of $d$ different $(t, n)$-threshold signature schemes is attempted. The timeout in use depends on the value $t$ (the number of different partial signatures required to compute a standard signature). For each unit increase of threshold $t$, the timeout increases by $\beta$ milliseconds.

Each of the $d$ signature schemes is tried in sequence until a complete signature is constructed or the timeout expires, so at most $d \times$ timeout milliseconds are spent on the extended group-based system.

3) If the extended group-based system does not work either, the semi-private protocol (see Section III-E) is tried.

1) Compound set-up phase: The compound protocol is composed of three schemes. In previous sections, we have presented the set-up phase for each of these schemes. We next explain the compound set-up phase when deploying such a system in a real environment.

Let us consider the co-existence of $m$ carmakers in a certain area. The $i$-th carmaker produces $v_{i}$ hundreds of thousands of vehicles per year. According to the European Environment Agency [24], the EU-15 area had about 170 millions of vehicles in 2004. Even though the carmakers produce $v_{1}+\cdots+v_{m}$ hundreds of thousands of new cars each year, there is also a large quantity of old vehicles which are eliminated in the same period. Therefore, the size of the vehicle fleet in a certain area does not undergo a strong increase from year to year. Value $n$ is the maximum number of vehicles allowable in the system covering the area. The only assumption on $n$ is that it cannot be greater than the cardinality of the group used to construct the BLS signature scheme. For cryptographic security reasons, this cardinality should be at least $2^{160}$. So, we can set a value for $n$ close to this upper limit. Such a huge $n$ ensures that we will never run out of key shares. As it can be seen in Section II a huge $n$ can be used without any negative impact on the system performance.

Our system requires a governmental authority $G A$ in the geographical area of deployment to ensure a correct set-up phase. Note that this authority is no longer needed when executing the compound protocol. The only role of the authority is to coordinate share distribution among the vehicles produced by different carmakers. In this way, $G A$ establishes $d$ signature schemes and the number $r$ of groups of vehicles in the area. According to that, each signature scheme generates $r$ shares. Each share is linked to one group. Additionally, $G A$ partitions the $n$ possible vehicles into several subranges, each of which is assigned to a carmaker. It also establishes $d^{\prime}$ different threshold signature schemes. Each one generates $n$ shares. Note that a certain carmaker receives the shares that correspond to its assigned subrange of $n$.

Now, let us consider that a certain vehicle $P_{i}$ is manufactured. This car has to be set up by its carmaker for both extended group-base and semi-private protocols. This process has been explained individually in Sections III-D and III-E. 
We next summarize it:

- Extended group-base protocol. For each signature scheme $k=1, \ldots, d, P_{i}$ is randomly assigned by the carmaker to a group $j_{k}$ (where $j_{k} \in\{1, \ldots, r\}$ ) and it is equipped with the share corresponding to group $j_{k}$.

- Semi-private protocol. $P_{i}$ is equipped with shares $\left(S K_{i}^{1}, \ldots, S K_{i}^{d^{\prime}}\right)$, where the share $S K_{i}^{w}$ is obtained by evaluating the polynomial of the $w$-th scheme at point $\alpha_{i}^{w}$, which is assumed to belong to the subrange of the carmaker of $P_{i}$.

The compound set-up phase we have presented relies on the assumption that an authority $G A$ exists which coordinates share distribution. An open problem is to devise a compound set-up phase which can work when no $G A$ is available.

\section{Simulation}

Our scheme for secure vehicle-generated announcements over VANETs was simulated in a realistic environment, where the range of car-to-car broadcasts was assumed to be $100 \mathrm{~m}$ (the worst-case, urban range according to [23]).

The goal of our simulations is to observe the performance of the compound protocol explained in Section III-F. This protocol requires a timeout that depends on values $\beta$ and $t$. In our simulations, we have fixed $\beta$ to $50 \mathrm{~ms}$. According to that, $t=4$ represents a timeout of 200 milliseconds.

\section{A. Simulation set-up}

The network simulator $n s-2$ [25] was used. The VANET scenario was built using the scenario generator presented in [26]. The road network considered covered an area of 2.4 $\mathrm{km}$ by $2.4 \mathrm{~km}$ and is shown in Figure 1.

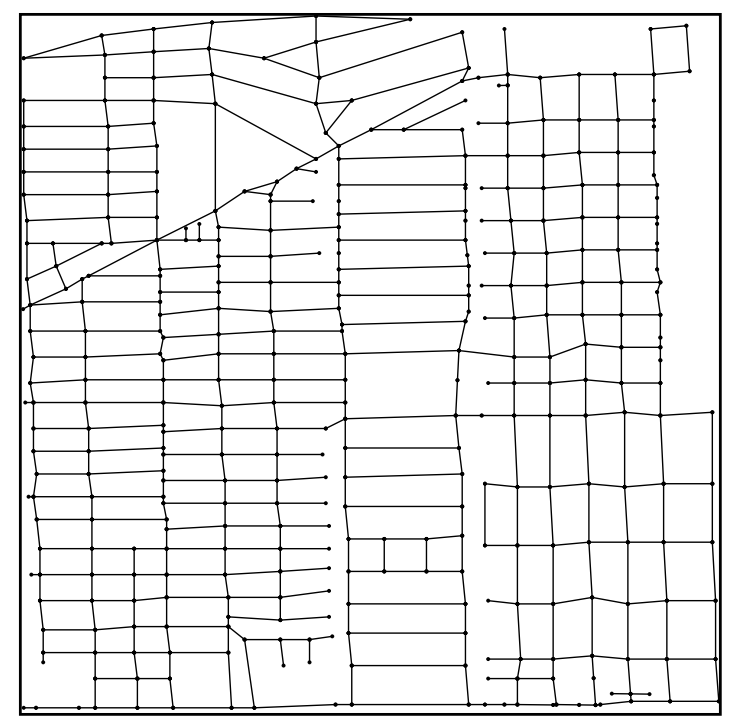

Fig. 1. Simulation scenario

In our simulations, the primary indicator examined is the probability for a certain announcement to get validated. An announcement is validated when its standard signature is constructed from $t$ different partial signatures generated by $t$ different cars. Those vehicles can belong to $t$ different groups or to only one group depending on whether the extended group-based protocol or the semi-private protocol are used.

A second indicator taken into account is the average number of different $(t, n)$-threshold signature schemes which are used when applying the extended group-based protocol. This indicator determines the time needed to validate the announcements.

Both indicators are essential to evaluate whether our scheme is usable in real VANETs.

In Section IV-B the optimal values for the parameters used in our system are studied. The goal of that study is to select the values for parameters based on the two indicators stated above for a wide range of vehicle densities. When the system is running, parameter values cannot be easily modified, so a parameter choice must be made which works well under several road conditions. The lessons learned from the simulations are summarized in Section IV-C.

The results given in what follows are average values obtained from 100 executions performed for each parameter choice.

\section{B. Parameter selection}

Vehicle density is expressed in vehicles $/ \mathrm{km}^{2}$. This value is changed by varying the total number of vehicles in the scenario represented in Figure 1.

Let $t$ stand for the minimum number of vehicles needed to validate an announcement. Each vehicle should belong to a different group when using the extended group-based protocol. Under the semi-private protocol there are no group constraints.

Table II shows the average probability $p_{1}$ of a certain announcement to be validated using the extended group-based protocol for fixed $r$ and several values of $t$. Value $i$ indicates the average number of different threshold signature schemes tried (each one is used until a timeout occurs) in order to validate the announcement with the extended group-based protocol. When the number of different threshold signature schemes tried for a certain announcement reaches the number $d$ of available schemes without the announcement being validated under any of them, the semi-private protocol is launched. We have set $d=3$ given the values $i$ obtained in preliminary simulations. This will be further explained below.

Value $p_{2}$ is the average probability of validating the announcement under the semi-private protocol when the extended group-based protocol fails. Note that the semi-private protocol is a tolerable fallback for low vehicle densities. For higher vehicle densities, the probability $p_{1}$ of successful validation with the extended group-based protocol is already very high, so that the instances in which the semi-private protocol is used as a fallback are very difficult ones (e.g. very sparse locations); this explains the near zero $p_{2}$ values for higher densities. Also, the N/A value for $p_{2}$ means that there was no need to call the semi-private protocol. We have set $d^{\prime}=20$ as the number of different threshold signature schemes available in the semi-private protocol; this should yield a good trade-off between unlinkability and implementation cost in the vehicles.

Results in Table II are given as a function of vehicle density and the minimum number of validating groups $t$. For this 
TABLE II

AVERAGE VALIDATION PROBABILITY $p_{1}$ AND AVERAGE NUMBER $i$ OF DIFFERENT THRESHOLD SIGNATURE SCHEMES TRIED FOR THE EXTENDED GROUP-BASED PROTOCOL, FOR CONSTANT NUMBER OF GROUPS $r=10$. AVERAGE VALIDATION PROBABILITY $p_{2}$ FOR THE SEMI-PRIVATE PROTOCOL WHEN THE EXTENDED GROUP-BASED PROTOCOL FAILS. RESULTS ARE GIVEN AS A FUNCTION OF VEHICLE DENSITY AND THE MINIMUM NUMBER OF VALIDATING VEHICLES $t$.

\begin{tabular}{|c|c|c|c|c|c|c|c|c|c|}
\hline Vehic. dens. & \multicolumn{3}{|c|}{$t=4$} & \multicolumn{3}{c|}{$t=5$} & \multicolumn{3}{|c|}{$t=6$} \\
\cline { 2 - 9 } & $p_{1}$ & $i$ & $p_{2}$ & $p_{1}$ & $i$ & $p_{2}$ & $p_{1}$ & $i$ & $p_{2}$ \\
\hline \hline 6.94 & 0.48 & 1.67 & 0.07 & 0.24 & 1.81 & 0.12 & 0.00 & N/A & 0.04 \\
8.68 & 0.64 & 1.69 & 0.02 & 0.33 & 1.74 & 0.05 & 0.04 & 2.00 & 0.04 \\
12.15 & 0.70 & 1.25 & 0.01 & 0.40 & 1.80 & 0.04 & 0.20 & 1.60 & 0.11 \\
15.62 & 0.72 & 1.22 & 0.00 & 0.44 & 1.70 & 0.04 & 0.36 & 1.85 & 0.10 \\
17.36 & 0.76 & 1.17 & 0.00 & 0.68 & 1.59 & 0.09 & 0.52 & 1.57 & 0.08 \\
24.31 & 0.94 & 1.14 & 0.00 & 0.76 & 1.53 & 0.02 & 0.72 & 1.67 & 0.11 \\
31.25 & 0.96 & 1.13 & 0.00 & 0.92 & 1.17 & 0.00 & 0.81 & 1.51 & 0.05 \\
38.19 & 0.96 & 1.09 & 0.00 & 0.94 & 1.08 & 0.00 & 0.89 & 1.48 & 0.00 \\
45.14 & 1.00 & 1.00 & N/A & 0.94 & 1.09 & 0.00 & 0.92 & 1.33 & 0.00 \\
52.08 & 1.00 & 1.00 & N/A & 1.00 & 1.00 & N/A & 0.96 & 1.26 & 0.00 \\
\hline
\end{tabular}

experiment, the number of groups of vehicles was set to $r=10$. The dependency on this value $r$ will be studied below, in Table III.

It can be observed in Table II that both validation probabilities $p_{1}$ and $p_{2}$ decrease as $t$ increases, no matter whether the VANET is sparse or dense. This is not surprising because validation is "easier" for smaller $t$; however, the price paid is that for smaller $t$ the trustworthiness of a validated message is lower. Following this argument, it is also expected that for very sparse networks (vehicle density of 6.94 ) and high $t$ values ( $t=6$ for instance) the extended group-based protocol is unable to validate a single announcement; in fact, not even the semi-private protocol works properly in that setting $\left(p_{2}=0.04\right.$ for a vehicle density of 6.94$)$. As a trade-off between trustworthiness and availability, it is suggested to take $t=4$ or $t=5$ depending on the desired trustworthiness level for the announcements. In fact, $t=5$ is the highest reasonable value because, even though $t=6$ works fine for dense VANETs (vehicle density above 38.19 ), it does not for medium-density $\left(p_{1}=0.52\right.$ for a density of 17.36$)$ and sparse VANETs. Since a threshold must be chosen which works properly under several road conditions, it is better to select $t<6$. In what follows, $t=4$ is taken.

Simulation shows that the average number $i$ of different threshold signature schemes tried by the extended group-based protocol decreases when the vehicle density increases and increases when the threshold $t$ increases. All in all, usually $i \leq 2$ whenever validation is successful, which is the usual outcome for medium- to high-density VANETs and moderate threshold $(t=4)$. For very sparse networks, validation mainly relies on the semi-private protocol so we can choose the number $d$ of signature schemes for the extended group-based protocol by considering only medium- to high-density VANETs. Thus a choice of $d=3$ is fair enough and is assumed in what follows; this implies that at most $3 \times$ timeout milliseconds are spent on the extended group-based protocol (as said above, for $t=4$ we consider timeout $=200$ milliseconds, so the overall time spent on the extended group-based protocol is $600 \mathrm{~ms}$ ).

Table III shows the average probability $p_{1}$ of a certain announcement being validated using the extended group-based protocol for fixed $t$ and several values of $r$. If this protocol fails, the semi-private fallback is launched. Value $p_{2}$ represents the average probability of validating a message with the semiprivate protocol when the extended group-based protocol fails. Finally, value $g$ represents the average group size. All results are given as a function of vehicle density and the number of groups of vehicles $r$. A value of $r$ must be set which works fine for very different vehicle densities. Also, $r$ must be chosen considering the trade-off between unlinkability and availability (see related discussion in Section III-C1): value $r$ should be greater than $t$ in order to guarantee availability (i.e., so that finding $t$ endorsing vehicles from different groups is easy). However, a big $r$ implies that the group size $g$ is small $(g:=n / r)$. In this way, the unlinkability of a certain vehicle is poor. In contrast, for a small $r$, the unlinkability of a certain vehicle is very high but the validation probability decreases. Table III reflects the availability problems of the system in very sparse VANETs when a certain unlinkability level is demanded. More specifically, we can observe that with a vehicle density of 6.94 and an average group size $g=5.0$ (which occurs when $r=8$ ), the probability $p_{1}$ of a certain announcement to be validated is 0.33 . Note that larger group sizes (which imply $r \ll 8$ ) will yield worse availability results. When availability problems arise, the system resorts to the semi-private protocol (which is less good in terms of privacy, unless $d^{\prime}$ is extremely high).

According to the above considerations, $r=10$ is taken as a reasonable trade-off between unlinkability and availability for all vehicle densities.

Note. As mentioned in Section III-C1, unlinkability is proportional to the group size $g$. One might object that the average group size in the simulations is small, which is true because the small geographical area considered $(2.4 \mathrm{~km}$ by $2.4 \mathrm{~km}$ ) can only accommodate a small number of vehicles. However, the purpose of the simulation is to evaluate the validation probability, which is independent of the group size (it only depends on the threshold $t$, the number of groups $r$ and the vehicle density). In a real scenario (e.g. the EU-15 area with 170 million vehicles mentioned in Section III-F1), the same $t$ and $r$ values used in the simulations can be employed, which will result in a very large group size $g$ guaranteeing high unlinkability. 
TABLE III

AVERAGE VALIDATION PROBABILITY $p_{1}$ FOR THE EXTENDED GROUP-BASED PROTOCOL AND AVERAGE VALIDATION PROBABILITY $p_{2}$ FOR THE SEMI-PRIVATE PROTOCOL WHEN THE EXTENDED GROUP-BASED PROTOCOL FAILS; THE AVERAGE GROUP SIZE $g$ IS SHOWN TOO. RESULTS ARE GIVEN AS A FUNCTION OF VEHICLE DENSITY AND NUMBER OF GROUPS $r$, FOR CONSTANT THRESHOLD $t=4$

\begin{tabular}{|c|c|c|c|c|c|c|c|c|c|c|c|c|c|}
\hline Vehic. dens. & \multicolumn{3}{|c|}{$r=8$} & \multicolumn{3}{c|}{$r=10$} & \multicolumn{3}{c|}{$r=15$} & \multicolumn{3}{c|}{$r=20$} \\
\cline { 2 - 11 } & $p_{1}$ & $p_{2}$ & $g$ & $p_{1}$ & $p_{2}$ & $g$ & $p_{1}$ & $p_{2}$ & $g$ & $p_{1}$ & $p_{2}$ & $g$ \\
\hline \hline 6.94 & 0.33 & 0.08 & 5.0 & 0.48 & 0.07 & 4.0 & 0.54 & 0.01 & 2.7 & 0.56 & 0.00 & 2.0 \\
8.68 & 0.38 & 0.05 & 6.2 & 0.64 & 0.02 & 5.0 & 0.66 & 0.03 & 3.3 & 0.66 & 0.00 & 2.5 \\
12.15 & 0.52 & 0.04 & 8.7 & 0.70 & 0.01 & 7.0 & 0.76 & 0.00 & 4.7 & 0.74 & 0.00 & 3.5 \\
15.62 & 0.67 & 0.00 & 11.2 & 0.72 & 0.00 & 9.0 & 0.76 & 0.00 & 6.0 & 0.88 & 0.00 & 4.5 \\
17.36 & 0.71 & 0.00 & 12.5 & 0.76 & 0.00 & 10.0 & 0.88 & 0.00 & 6.7 & 0.93 & 0.00 & 5.0 \\
24.31 & 0.86 & 0.00 & 17.5 & 0.94 & 0.00 & 14.0 & 0.96 & 0.00 & 9.3 & 0.99 & 0.00 & 7.0 \\
31.25 & 0.93 & 0.00 & 22.5 & 0.96 & 0.00 & 18.0 & 1.00 & N/A & 12.0 & 1.00 & N/A & 9.0 \\
38.19 & 0.94 & 0.00 & 27.5 & 0.96 & 0.00 & 22.0 & 1.00 & N/A & 14.7 & 1.00 & N/A & 11.0 \\
45.14 & 0.97 & 0.00 & 32.5 & 1.00 & N/A & 26.0 & 1.00 & N/A & 17.3 & 1.00 & N/A & 13.0 \\
52.08 & 1.00 & N/A & 37.5 & 1.00 & N/A & 30.0 & 1.00 & N/A & 20.0 & 1.00 & N/A & 15.0 \\
\hline
\end{tabular}

\section{Lessons learned from the simulation}

The probability of successful validation depends on the threshold $t$, the number $r$ of groups and the vehicle density, regardless of the group size. For a fixed density, the greater $r$ with respect to $t$, the higher the success probability. The closer $r$ to $t$, the lower the success probability.

All simulations performed reflect that with the parameter selection used ( $t=4, r=10$ and $d=3$ ), our proposal provides message trustworthiness and vehicle unlinkability under different road conditions. Results show that our scheme performs best in medium- to high-density VANETs (densities from 12.15 to 52.08 ). Nevertheless, it works fair enough in very sparse environments as well:

- For a vehicle density 6.94, our scheme achieves a success probability $p_{1}=0.48$ in announcement validation with the extended group-based protocol. In the cases when this protocol fails, the semi-private one works with a probability $p_{2}=0.07$.

- For a vehicle density 8.68 , the success probability with the extended group-based protocol increases to $p_{1}=$ 0.64 . The semi-private protocol used as a fallback earns an additional $p_{2}=0.02$.

The low success in validation for sparse VANETs should be put in context: in an area with very low traffic, it is often less critical to get announcements on road conditions, as there is hardly anyone who can benefit from them.

\section{CONCLUSION}

In this paper, a new system has been presented for secure vehicle-generated announcements on VANETs that relies on a priori measures against internal attackers (vehicles in the VANET sending fake messages). Thanks to the use of threshold signatures, our system outperforms previous proposals in message length and computational cost.

However, it would be very unfair if vehicles and drivers volunteering to co-operate in generating trustworthy announcements could be tracked and saw their privacy diminished. Three different variants of the system have been proposed to achieve unlinkability without losing trustworthiness: the first variant is a special case of the second one and is better suited to dense VANETs, whereas the second and third variants can be used as fallbacks for sparse VANETs. This naturally leads to combining the three variants in a compound protocol.

\section{DISCLAIMER AND ACKNOWLEDGMENTS}

Thanks go to Úrsula González-Nicolás for programming the required simulations. The authors are with the UNESCO Chair in Data Privacy, but they are solely responsible for the views expressed in this paper, which do not necessarily reflect the position of UNESCO nor commit that organization. This work was partly supported by the Spanish Government through projects TSI2007-65406-C03-01 "E-AEGIS" and CONSOLIDER INGENIO 2010 CSD200700004 "ARES", and by the Government of Catalonia under grant 2005 SGR 00446.

\section{REFERENCES}

[1] F. Dötzer, "Privacy issues in vehicular ad hoc networks", Lecture Notes in Computer Science, vol. 3856, pp. 197-209, 2006.

[2] M. Raya, P. Papadimitratos, and J.-P. Hubaux, "Securing vehicular communications", IEEE Wireless Communications Magazine, vol. 13, no. 5, pp. 8-15, 2006.

[3] M. Raya and J.-P. Hubaux, "Securing vehicular ad hoc networks", Journal of Computer Security, Special Issue on Security of Ad Hoc and Sensor Networks, vol. 15, no. 1, pp. 39-68, 2007.

[4] M. Raya, P. Papadimitratos, I. Aad, D. Jungels and J.-P. Hubaux, "Eviction of misbehaving and faulty nodes in vehicular networks", IEEE Journal on Selected Areas in Communications, vol. 25, no. 8, pp. 1557$1568,2007$.

[5] F. Armknecht, A. Festag, D. Westhoff and K. Zeng, "Cross-layer privacy enhancement and non-repudiation in vehicular communication", in 4th Workshop on Mobile Ad-Hoc Networks (WMAN), Bern, Switzerland, March 2007.

[6] J. Guo, J.P. Baugh and S. Wang, "A group signature based secure and privacy-preserving vehicular communication framework", in Mobile Networking for Vehicular Environments, pp. 103-108, 2007.

[7] X. Lin, X. Sun, P.-H. Ho and X. Shen, "GSIS: A secure and privacy preserving protocol for vehicular communications", IEEE Transactions on Vehicular Technology, vol. 56, no. 6, pp. 3442-3456, 2007.

[8] C. Gamage, B. Gras and A.S. Tanenbaum, "An identity-based ring signature scheme with enhanced privacy", in Proceedings of the IEEE SecureComm Conference, pp. 1-5, 2006.

[9] P. Golle, D. Greene and J. Staddon, "Detecting and correcting malicious data in VANETs", in Proceedings of the 1st ACM international workshop on Vehicular Ad Hoc Networks, pp. 29-37, 2004.

[10] B. Parno and A. Perrig, "Challenges in securing vehicular networks", in Proceedings of the ACM Workshop on Hot Topics in Networks, 2005.

[11] B. Ostermaier, F. Dötzer and M. Strassberger, "Enhancing the security of local danger warnings in VANETs - A simulative analysis of voting schemes", in Proceedings of the The Second International Conference on Availability, Reliability and Security, pp. 422-431, 2007.

[12] M. Raya, A. Aziz and J.-P. Hubaux, "Efficient secure aggregation in VANETs", in Proceedings of the 3rd International Workshop on Vehicular Ad hoc Networks - VANET'06, pp. 67-75, 2006.

[13] A. Shamir, "How to share a secret", Communications of the ACM, vol. 22, pp. 612-613, 1979. 
[14] V. Shoup, "Practical threshold signatures", in Advances in Cryptology - Eurocrypt'00, Lecture Notes in Computer Science, vol. 1807, pp. 207220, 2000.

[15] A. Boldyreva, "Threshold signatures, multisignatures and blind signatures based on the Gap-Diffie-Hellman-group signature scheme", in Public Key Cryptography 2003, Lecture Notes in Computer Science, vol. 2567, pp. 31-46, 2003.

[16] P. A. Fouque and J. Stern, "Fully distributed threshold RSA under standard assumptions", in Proceedings of Asiacrypt'01, Lecture Notes in Computer Science, vol. 2248, pp. 310-330, 2001.

[17] I. Damgård and M. Koprowski, "Practical threshold RSA signatures without a trusted dealer", in Advances in Cryptology-Eurocrypt'01, Lecture Notes in Computer Science, vol. 2045, pp. 152-165, 2001.

[18] D. Boneh, B. Lynn, and H. Shacham, "Short signatures from the Weil pairing", in Advances in Cryptology-Asiacrypt 2001, Lecture Notes in Computer Science, vol. 2248, pp. 514-532, 2001.

[19] P. S. L. M. Barreto, H. Y. Kim, B. Lynn, and M. Scott, "Efficient algorithms for pairing-based cryptosystems", in Advances in Cryptology - Crypto'2002, Lecture Notes in Computer Science, vol. 2442, pp. 354 368, 2002.

[20] R. Gennaro, S. Jarecki, H. Krawczyk and T. Rabin, "Robust threshold DSS signatures", in Advances in Cryptology - Eurocrypt'96, Lecture Notes in Computer Science, vol. 1070, pp. 354-371, 1996.

[21] C. Blundo and D. R. Stinson, "Anonymous secret sharing schemes", Discrete Applied Mathematics, vol. 77, pp. 13-28, 1997.

[22] N. M. Rabadi and S. M. Mahmud, "Performance evaluation of IEEE 802.11a MAC protocol for vehicle intersection collision avoidance system", in Consumer Communications and Networking Conference - CCNC 2007, pp. 54-58, 2007.

[23] I. Berger, "Standards for car talk", IEEE The Institute, vol. 31, no. 1, Mar. 2007.

[24] European Environment Agency, http://www.eea.europa.eu

[25] The Network Simulator - ns, http://nsnam.isi.edu/nsnam/index.php/Main_Page

[26] A. K. Saha and D. B. Johnson, "Modeling mobility for vehicular ad hoc networks", in Proceedings of the 1st International Workshop on Vehicular Ad Hoc Networks-VANET'2004, ACM: Philadelphia, USA, pp. 91-92, 2004. 